\title{
Microfluidics for rapid detection of isocitrate dehydrogenase 1 mutation for intraoperative application
}

\author{
*Abudumijiti Aibaidula, MD, ${ }^{1}$ Wang Zhao, PhD, ${ }^{2}$ Jin-song Wu, MD, PhD, ${ }^{1}$ Hong Chen, MD, ${ }^{3}$ \\ Zhi-feng Shi, MD, ${ }^{1}$ Lu-lu Zheng, PhD, ${ }^{2}$ Ying Mao, MD, PhD, ${ }^{1}$ Liang-fu Zhou, MD, PhD, and \\ Guo-dong Sui, PhD²
}

${ }^{1}$ Glioma Surgery Division, Neurological Surgery Department, Huashan Hospital, ${ }^{2}$ Department of Environmental Science and Engineering, Institute of Biomedical Sciences, and ${ }^{3}$ Department of Neuropathology, Huashan Hospital, Shanghai Medical College, Fudan University, Shanghai, China

\begin{abstract}
OBJECTIVE Conventional methods for isocitrate dehydrogenase 1 (IDH1) detection, such as DNA sequencing and immunohistochemistry, are time- and labor-consuming and cannot be applied for intraoperative analysis. To develop a new approach for rapid analysis of IDH1 mutation from tiny tumor samples, this study used microfluidics as a method for IDH1 mutation detection.

METHODS Forty-seven glioma tumor samples were used; IDH1 mutation status was investigated by immunohistochemistry and DNA sequencing. The microfluidic device was fabricated from polydimethylsiloxane following standard soft lithography. The immunoanalysis was conducted in the microfluidic chip. Fluorescence images of the on-chip microcolumn taken by the charge-coupled device camera were collected as the analytical results readout. Fluorescence signals were analyzed by NIS-Elements software to gather detailed information about the IDH1 concentration in the tissue samples.
\end{abstract}

RESULTS DNA sequencing identified IDH1 R132H mutation in 33 of 47 tumor samples. The fluorescence signal for IDH1-mutant samples was $5.49 \pm 1.87$ compared with $3.90 \pm 1.33$ for wild type $(p=0.005)$. Thus, microfluidics was capable of distinguishing IDH1-mutant tumor samples from wild-type samples. When the cutoff value was 4.11 , the sensitivity of microfluidics was $87.9 \%$ and the specificity was $64.3 \%$.

CONCLUSIONS This new approach was capable of analyzing IDH1 mutation status of tiny tissue samples within 30 minutes using intraoperative microsampling. This approach might also be applied for rapid pathological diagnosis of diffuse gliomas, thus guiding personalized resection.

http://thejns.org/doi/abs/10.3171/2015.4.JNS141833

KEY WORDS isocitrate dehydrogenase 1; microfluidics; neurosurgery; glioma; oncology

$\mathrm{S}$ EQUENCE analysis of glioblastoma found a mutation in isocitrate dehydrogenase 1 (IDH1), which mostly resulted in the replacement of wild-type arginine in position 132 with histidine $(\mathrm{R} 132 \mathrm{H}) .{ }^{25}$ Studies have reported that the $I D H 1$ mutation is frequently detected in diffuse astrocytoma $(59 \%-88 \%)$, anaplastic astrocytoma $(52 \%-78 \%)$, oligodendroglioma $(68 \%-82 \%)$, anaplastic oligodendroglioma $(60 \%-75 \%)$, oligoastrocytoma (50\%-94\%), anaplastic oligoastrocytoma (66.1\%-78\%), and secondary glioblastomas $(50 \%-88 \%)$, but rarely in primary glioblastomas $(3 \%-7 \%){ }^{1}{ }^{114} \mathrm{IDHI}$ mutation was also closely associated with young age and better clinical outcome. ${ }^{34}$

Currently, the detection of IDHI mutation status can be achieved by DNA-based methods, such as DNA pyrosequencing ${ }^{10}$ polymerase chain reaction (PCR) ${ }^{13,24}$ and immunohistochemistry (IHC). ${ }^{5}$ The majority of laboratories use IHC for initial screening for IDHI mutation and, if results are negative, use DNA sequencing for less common IDHI mutant types. DNA sequencing provides direct

ABBREVIATIONS AUC = area under the curve; FFPE = formalin-fixed paraffin-embedded; IDH1 = isocitrate dehydrogenase 1 ; IHC = immunohistochemistry; PCR = polymerase chain reaction; $\mathrm{ROC}=$ receiver operating characteristic.

SUBMITTED August 8, 2014. ACCEPTED April 30, 2015.

INCLUDE WHEN CITING Published online November 6, 2015; DOI: 10.3171/2015.4.JNS141833.

* Drs. Aibaidula and Zhao contributed equally to this work. 
evidence for $I D H I$ gene mutation. However, it is difficult to detect $I D H I$ mutation in highly contaminated tumor samples, and enrichment methods are needed..$^{32}$

Moreover, elaborate laboratory equipment for DNA sequencing or PCR is not available in most neuropathology settings. Anti-IDH1 R132H immunostaining based on commercially available antibody has been confirmed as a reliable method for the detection of $I D H 1$ mutation. ${ }^{27}$ For the IDHI RI32H mutation type, both its sensitivity and specificity are up to $100 \% .{ }^{20}$ IHC may be more sensitive than sequencing, especially for tiny biopsy samples. ${ }^{27,32}$ IHC can also be used to identify a single infiltrated tumor cell and discriminate the infiltrating edge of IDHI-mutant tumors from reactive gliosis. ${ }^{4,5}$ Above all, a recent study indicated that IDHI mutation in malignant astrocytoma could serve as a predictive molecular biomarker to guide extensive resection, allowing for individualized therapy based on tumor genotype. ${ }^{3}$ However, regular IHC takes more than 48 hours and cannot meet the demand of intraoperative analysis.

Microfluidics, also known as lab-on-a-chip, is a technique that manipulates or processes small volumes of fluids at micron dimension. ${ }^{2,33}$ It is attracting growing attention in many fields because it requires less time and less reagent. Other advantages include rapid reaction speed, easy control of reaction conditions, and easy fabrication. ${ }^{8,18,31}$ It can also integrate microvalves with pumps to precisely control the reagent inside the microchips, which is perfect for performing complicated biomarker analysis from a tiny sample within a short period of time. .,11,29,30 $^{2}$ Herein we describe a new approach for rapid analysis of IDH1 mutation from microsampling of tissues, which was successfully developed using a microfluidic device. This new approach is capable of rapid analysis of $\mathrm{IDHI}$ mutation status from tiny amounts of tissue within 30 minutes, using much less sample and smaller reagent amounts compared with conventional methods..$^{11,15,35}$

\section{Methods}

\section{Patients and Tissue Specimens}

Forty-seven glioma tumor samples were obtained from the Glioma Tissue Bank of the Glioma Surgery Division, Neurological Surgery Department of Huashan Hospital, Fudan University. All specimens were prospectively collected in the operating room, in accordance with protocols approved by Huashan Institutional Review Board, between 2012 and 2013. Each patient gave informed consent before the operation. For microfluidics, each retrieved frozen sample was around $5 \mathrm{mg}$. The retrieved samples were used in the experiment without purification. The diagnosis was made according to the histological assessments, following the WHO classification. ${ }^{19}$

\section{Microfluidics}

Reagents

Reagents used included ProteoExtract Complete Mammalian Proteome Extraction Kit (Cat. No. 539779, Calbiochem); anti-human IDH1 R132H (mouse monoclonal, Histonova); anti-human IDH1 (rabbit polyclonal, Epitomics); and FITC anti-mouse antibody (Abcam, Ltd).
Microspheres (Protein A, 9-micron diameter) were purchased from Bang's Laboratory. All other reagents (analytical purity) were purchased from Sigma.

\section{Microfluidic Device Fabrication}

The microfluidic device used for this study was fabricated from polydimethylsiloxane following standard soft lithography. The device was composed of 2 layers: upper fluidic and bottom control. Two different molds with different patterns were fabricated by standard soft lithographic processes. ${ }^{8,18,22,35}$ The structure of the proposed microfluidic chip is shown in Supplemental Fig. 1 and discussed in Supplemental Methods.

\section{Immunoanalysis on the Microfluidic Device}

Figure 1 shows the immunoanalysis mechanism within the microfluidic chip. The microspheres immobilized with anti-IDH1 polyclonal antibody were prepared according to the protocols from Bang's Laboratory. The microspheres were put into the chip to form the microcolumn before the application. During the analysis process, the tiny tumor sample collected by needle tip was directly injected into the lysis chamber. The lysis process started with the ProteoExtract buffer being loaded into the chamber, which normally took 10 minutes. The lysis extraction solution (containing the residues) was loaded into the microcolumn and the residue was subsequently filtered off at the beginning of the microcolumn where it was filled with microspheres. When the protein extraction containing IDH1 and other proteins entered the column, only IDH1 became immobilized onto the column via binding between IDH1 and anti-IDH1 polyclonal antibody on the surface of the microspheres. Block buffer was then introduced for about 5 minutes, followed by loading of phosphate-buffered saline to wash away components that were not immobilized. After that, the buffer containing monoclonal antibody to IDH1 R132H was loaded into the column to complete the reaction. After 5 minutes, the column was washed with cleaning buffer. Afterward, the FITC anti-mouse secondary antibody solution was added into the column. Fluorescence images of the microcolumn were taken by a charge-coupled device camera equipped with a microscope. Fluorescence intensity was analyzed by NIS-Elements (Nikon) software for detailed information about the IDH1 concentration in the tissue samples.

In our study, DNA sequencing, IHC procedure, and slide analysis were performed by investigators who were blinded to the clinical information. For microfluidics, 2 investigators who were blinded to clinical information did the chip analysis.

\section{Immunohistochemistry of IDH1 R132H}

The EnVision FLEX detection system (Dako) and antiIDH1 R132H (Hu) from mouse (Clone: H09) (Dianova) were used. An automated immunostaining procedure was performed according to the Dianova technical note. Immunostaining was interpreted as positive when tumor nuclei showed strong positive staining for IDH1 R132H.

To precisely quantitate $\mathrm{IDH} I$-mutant protein expression in tumor cells, slides were further analyzed with the Vec- 


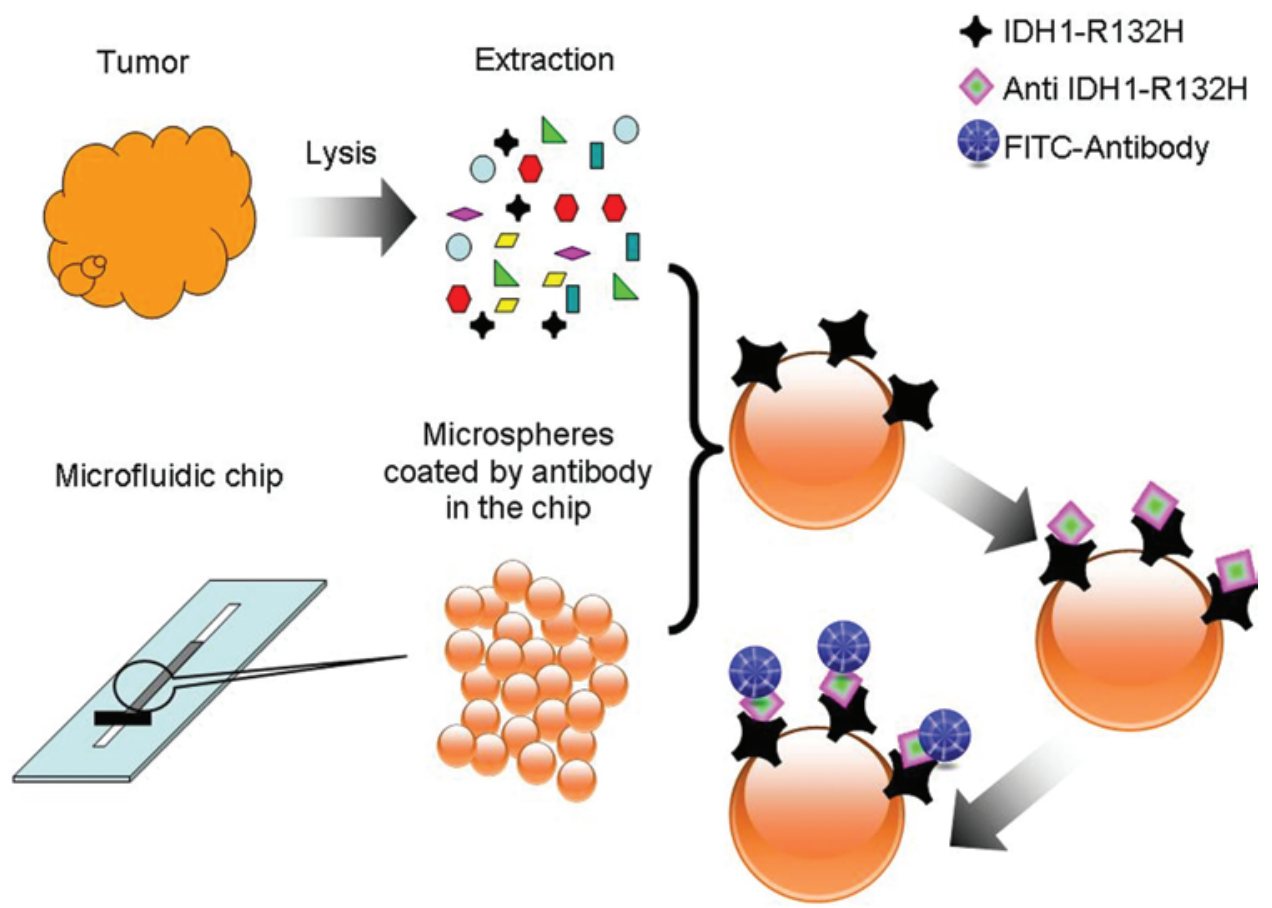

FIG. 1. A schematic illustration of the immunoanalysis mechanism. FITC = fluorescein isothiocyanate. Figure is available in color online only.

tra Automated Multispectral Imaging System equipped with inForm software (PerkinElmer, Inc.). Automated acquisition was in a batch run involving $\times 40$ whole-slide scans followed by high-magnification $(\times 200)$ imaging for regions of interest only. Histological images were digitized and then analyzed with the nuclear algorithm. Scores were generated using the inForm software, which provided the intensity of staining. For each slide, 5 high-magnification $(\times 200)$ images were obtained and analyzed separately. The average intensity score for nucleic regions of 5 images was considered as immunostaining intensity for each sample.

\section{DNA Sequencing of IDH1 R132H}

Formalin-fixed paraffin-embedded (FFPE) tumor samples were used. Two 10- $\mu$ m-thick slides of each sample were prepared. Tissue was microdissected from 2 FFPE sections to guarantee that tumor content was greater than $70 \%$. The primers of IDHI RI32 hotspot mutation were forward 5'-CGGTCTTCAGAGAAGCCATT-3' and reverse 5'-CACATTATTGCCAACATGAC-3'. A detailed description of the DNA sequencing process is given in Supplemental Methods.

\section{Statistical Analysis}

Statistical analysis was performed using Statistical Product and Service Solutions software (SPSS 20, IBM Corp.) and ROCR package in R (http://www.r-project. org). Fluorescence signals and intensity score difference between IDHI mutant and wild type was compared using the 2-sample Wilcoxon rank-sum test. Fluorescence intensity and the intensity score between different tumor types were compared using the Kruskal-Wallis test. The cutoff value and receiver operating characteristic (ROC) curve were calculated using the ROCR package in R. All tests were 2-tailed, with a CI of $95 \%$. A p value less than 0.05 was considered statistically significant.

\section{Results}

\section{Sample Characteristics}

Basic information about the 47 samples is shown in Supplemental Fig. 2. The subjects comprised 10 diffuse astrocytomas, 12 oligodendrogliomas, 8 anaplastic astrocytomas, 7 anaplastic oligodendrogliomas, and 10 primary glioblastomas. Samples of 22 male and 25 female patients were included, and the mean age was $45.70 \pm 12.64$ years. WHO grading revealed 22 Grade II samples, 15 Grade III, and 10 Grade IV.

\section{Microfluidics}

Figure 2 shows examples of fluorescence images of the on-chip microcolumns and corresponding IHC images for various tumor types. Differences between fluorescence images of microcolumns responding to various types of glioma could be easily identified. The shining spots on the images indicated the inhomogeneity of the lysis extract. The average intensity of the fluorescence images was collected and data analyses were executed to evaluate the performance of the proposed microfluidic device.

The fluorescence signal for $I D H 1$-mutant samples was $5.49 \pm 1.87$ compared with $3.90 \pm 1.33$ for wild type $(\mathrm{p}=$ $0.005)$. Thus, microfluidics was capable of distinguishing IDH1-mutant samples from wild type. Fluorescent signals for different tumor types are shown in Table 1. Comparison of fluorescence signals between different tumor types revealed statistical significance between primary 

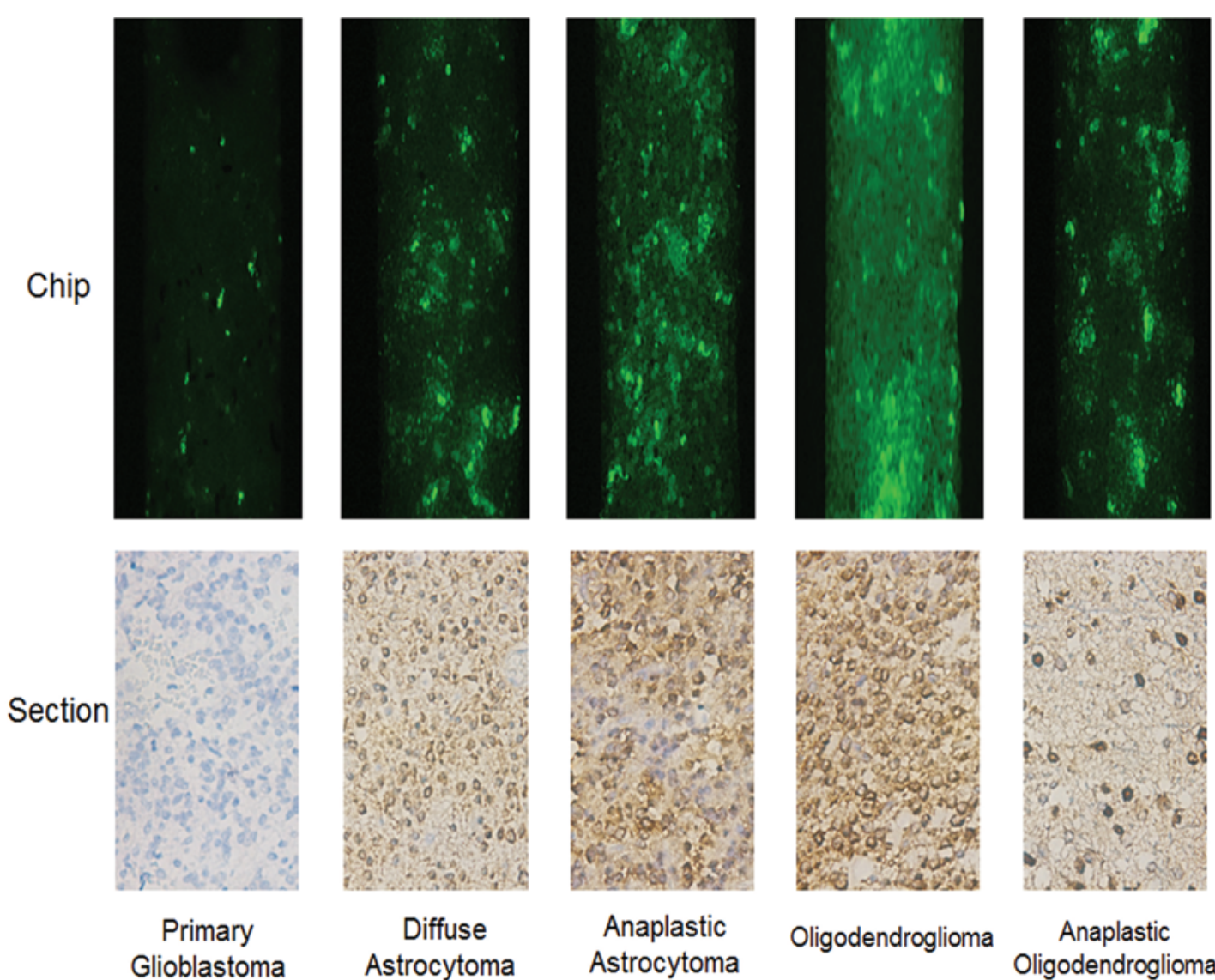

IDH1

$(-)$

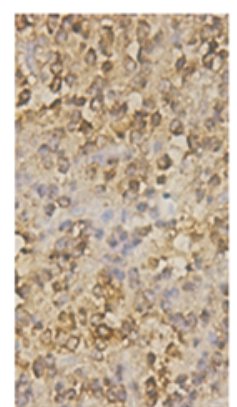

Anaplastic Astrocytoma

(+)

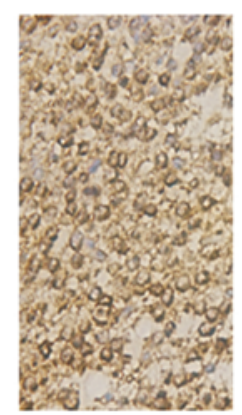

Oligodendroglioma

$(+)$

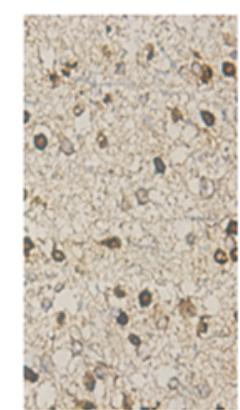

Anaplastic

Oligodendroglioma

$(-)$

FIG. 2. Fluorescence images of the on-chip microcolumns and IHC images for various tumor types. Fluorescence images of microcolumns and corresponding $\mathrm{IHC}$ images of 5 tumor types are shown. Original magnification $\times 200$. Figure is available in color online only.

glioblastoma and anaplastic astrocytoma (3.15 vs 5.57 , p $=0.011)$, oligodendroglioma ( 3.15 vs $5.14, \mathrm{p}=0.004)$, and diffuse astrocytoma ( 3.15 vs $6.36, \mathrm{p}<0.001)$. No statistical significance was observed between other tumor types. Each measurement was repeated 3 times and the average number was presented. The SD values were within the acceptable range, suggesting the feasibility of this approach to analyze the IDHI mutation status from tiny (about 5 $\mathrm{mg}$ ) samples. The whole process, from injecting samples to fluorescent signal readout, can be completed within 30 minutes, which is fast enough to perform during the surgical procedure. Figure 3 presents the distribution of all fluorescence signals. When the cutoff value was 4.11, it helped to distinguish most of the mutant samples from wild-type ones. Specificity and sensitivity are presented in Fig. 4.

\section{Detection of IDH1 R132H Mutation Status}

Mutational hotspot arginine at codon 132 of IDHI was evaluated by direct sequencing (the gold standard for gene mutation) and IHC (the routinely applied method in our clinical setting). Immunostaining was interpreted as positive when tumor cell nuclei showed strong positive staining for IDH1 R132H. The identical results of these 2 methods, presented in Table 1, confirmed that IHC was a reliable approach. Fluorescent signals of the tumor samples, as well as the IHC and DNA sequencing results, are presented in Table 1. DNA sequencing identified IDHI $R 132 \mathrm{H}$ mutation in 8 of 10 diffuse astrocytomas, 11 of 12 oligodendrogliomas, 8 of 8 anaplastic astrocytomas, 4 of 7 anaplastic oligodendrogliomas, and 2 of 10 primary glioblastomas. Slide analysis using inForm software revealed that the average intensity score of IHC-mutant samples was $0.151 \pm 0.069$ compared with $0.029 \pm 0.021$ for wild type $(\mathrm{p}<0.001)$. The intensity score of IHC for different tumor types is also presented in Table 1. Statistical significance was observed between primary glioblastoma and anaplastic astrocytoma ( 0.067 vs $0.161, \mathrm{p}=0.036)$, but not between primary glioblastoma and anaplastic oligodendroglioma $(0.067$ vs $0.091, \mathrm{p}=1.000)$, primary glioblastoma and diffuse astrocytoma (0.067 vs $0.110, \mathrm{p}=1.000$ ), primary glioblastoma and oligodendroglioma (0.067 vs $0.139, \mathrm{p}=0.337$ ), or between other different tumor types. Conventional IHC could be applied to detect the IDHI mutation status. However, this semiquantitative method could not be used to compare the protein levels of different tumor types. 


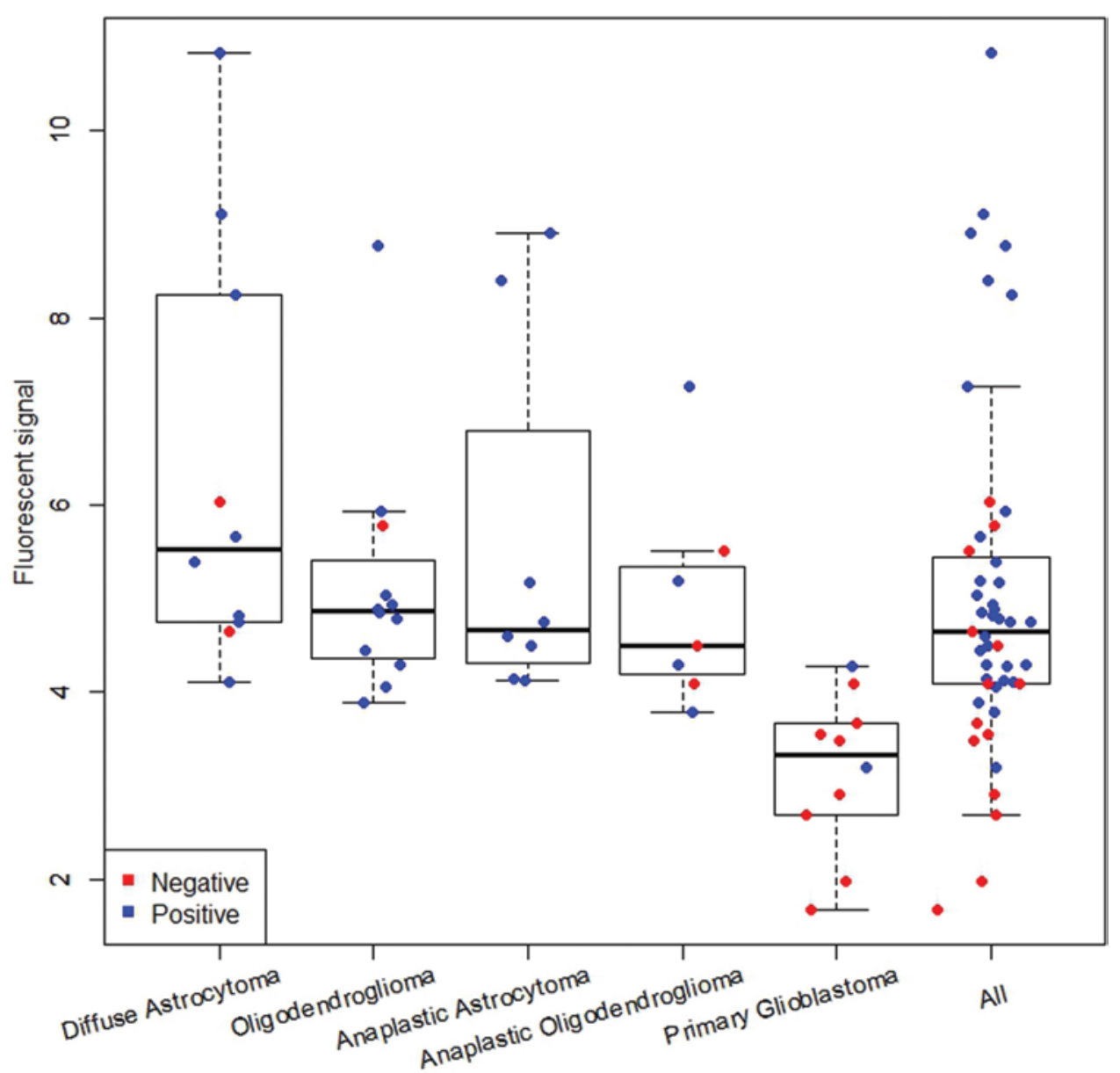

FIG. 3. Distribution of fluorescence signals for all tumor samples. It is clearly illustrated that there were discriminable differences in fluorescence signals between mutant and wild-type samples. The cutoff value was calculated using the ROCR package in $R$ (http://www.r-project.org/). When the cutoff value was 4.11, it helped to distinguish most of the mutant samples from wild-type samples. Fluorescent signals lower than the cutoff value were counted as wild-type samples in the microfluidics approach. Fluorescent signals higher than the cutoff value were counted as mutant samples in the microfluidics approach. Figure is available in color online only.

\section{Comparison of IHC and Microfluidics}

Both IHC and microfluidics showed the potential to detect IDHI mutation status. For comparison purposes, IHC images of these tumor samples were digitized and are presented in Table 1. The ROC curve was also established and the area under the curve (AUC) for microfluidics was 0.7608 (Fig. 4). When the cutoff value was 4.11, the sensitivity of microfluidics was $87.9 \%$ and the specificity was $64.3 \%$ (Fig. 4). During the IHC process, the FFPE tumor sample was attached with monoclonal antibody to IDH1 $\mathrm{R} 132 \mathrm{H}$, and stained by the second antibody at visible light range. In this procedure, the polyclonal anti-human IDH1 was not used, as compared with the immunoanalysis procedure using microfluidics. Whereas IHC focused more on the morphological information of the IDH1 distribution, the proposed microfluidics method emphasized the precise measurement of IDH1 expression from the tissue extract, yet could not provide any morphological information. Although a proportional linear relationship between the results from IHC and those from the proposed micro- fluidics approach was not expected, the comparison revealed that similar amounts of IDH1 were measured.

\section{Discussion \\ Feasibility of Microfluidics}

IDH1 mutation has been identified as a diagnostic and prognostic biomarker in glioma. ${ }^{12,21}$ Patients with malignant astrocytoma with $\mathrm{IDH} 1$ mutations have nearly twice as long, or even 10 times longer, median overall survival compared with those without mutations. ${ }^{3,34}$ A recent study also revealed that refinement of the classification of the 3 most common types of brain tumors could be achieved by telomerase reverse transcriptase promoter and $I D H 1 \mathrm{mu}-$ tation status. ${ }^{16}$ Mutation in the $I D H I$ gene mostly affects IDH1 protein, by replacing wild-type arginine in position 132 with histidine (R132H). This makes it feasible to detect IDHI gene mutation through IHC by using specific antibodies that bind mutant IDH1 protein. ${ }^{25}$ However, regular IHC analysis can only be applied for postoperative analysis. Preoperative precise detection of 2-hydroxyglutarate 


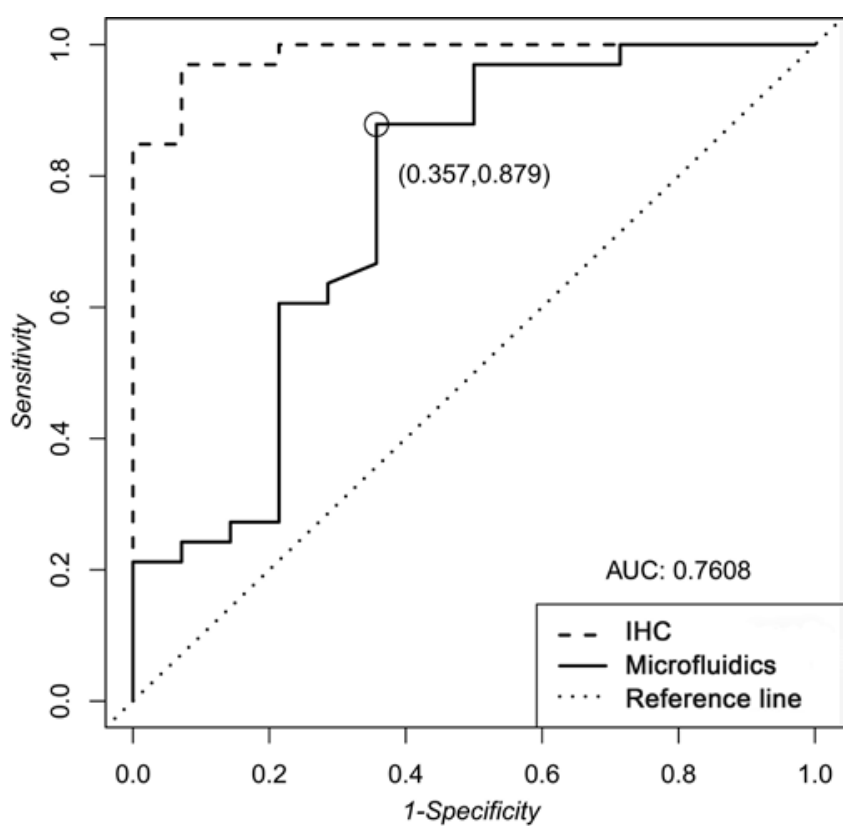

FIG. 4. The ROC curve for comparison of $\mathrm{IHC}$ and microfluidics. The AUC for microfluidics was 0.7608 . When the cutoff value was 4.11 , the sensitivity of microfluidics was $87.9 \%$ and the specificity was $64.3 \%$.

by MR spectroscopy is still under investigation., ${ }^{7,9,17,26}$ Our study, which is based on microfluidics and use of specific antibodies for $I D H 1$-mutant protein, was also applicable for detection of $I D H 1$ mutation status.

According to our statistical results, 4.11 was set as a cutoff value to distinguish most of the IDH1-mutant samples from wild type, regardless of the tumor type and grade. The microfluidic approach was developed to detect IDHI mutation status intraoperatively to guide personalized sur- gery; in other words, for use when the pathology result was not available or there was only a preliminary result obtained from frozen sections. Setting different cutoff values for different tumor types was not practical when it was applied during surgery, and it could even be confusing. When the cutoff value was 4.11 , the sensitivity of microfluidics was $87.9 \%$ and the specificity was $64.3 \%$. The low specificity was due to several aspects. First, from Table 1, we can see that different tumor types had different baselines for negative samples. This difference may come from manual operations, such as fluorescence image reading and sample dissection. Tumor inhomogeneity may also contribute to the different negative baselines, even within the same tumor type. We believe that in future experiments, after we modify our sample-input techniques and apply this method to more samples, the baseline deviation can be decreased. After integrating clinical information (e.g., patient age, course of disease, medical images, and frozen-section results), the final specificity will increase.

\section{Clinical Implications for Individualized Surgery}

Microfluidics is capable of intraoperative analysis of IDH1 mutation status, and the clinical implication lies in intraoperatively directing the individualized surgery. The chief implications are as follows.

1) For low-grade glioma, due to the lack of macroscopic discriminable tumor margin, the frozen section is the method for intraoperative identification of tumor infiltration. However, this method may not precisely identify the tumor infiltration from reactive gliosis because detectability mostly depends on the morphological experience of the neuropathologists. Thus, the possibility of misdiagnosis always exists. Because IDHI mutation has been identified as a Level IIb diagnostic biomarker, microfluidics can complement the conventional intraoperative pathological

TABLE 1. Fluorescence signal and IHC intensity score of different tumor types

\begin{tabular}{|c|c|c|c|c|c|}
\hline \multirow[b]{2}{*}{ Tumor Type } & \multirow[b]{2}{*}{ IDH1 R132H Mutation Status* } & \multicolumn{2}{|c|}{ Fluorescence Signal } & \multicolumn{2}{|c|}{$\mathrm{IHC}$ Intensity Score } \\
\hline & & Mean & $\mathrm{SD}$ & Mean & SD \\
\hline \multirow[t]{3}{*}{ Diffuse astrocytoma } & Negative $(n=2) ;$ wild type $(n=2)$ & 5.35 & 0.98 & 0.037 & 0.015 \\
\hline & Positive $(n=8) ;$ mutant type $(n=8)$ & 6.61 & 2.45 & 0.128 & 0.049 \\
\hline & All; all & 6.36 & 2.25 & 0.110 & 0.058 \\
\hline \multirow[t]{3}{*}{ Oligodendroglioma } & Negative $(n=1) ;$ wild type $(n=1)$ & 5.78 & NA & 0.026 & NA \\
\hline & Positive $(n=11) ;$ mutant type $(n=11)$ & 5.08 & 1.34 & 0.150 & 0.089 \\
\hline & All; all & 5.14 & 1.30 & 0.139 & 0.092 \\
\hline \multirow[t]{3}{*}{ Anaplastic astrocytoma } & Negative $(n=0) ;$ wild type $(n=0)$ & NA & NA & NA & NA \\
\hline & Positive $(n=8) ;$ mutant type $(n=8)$ & 5.57 & 1.93 & 0.161 & 0.057 \\
\hline & All; all & 5.57 & 1.93 & 0.161 & 0.057 \\
\hline \multirow[t]{3}{*}{ Anaplastic oligodendroglioma } & Negative $(n=3) ;$ wild type $(n=3)$ & 4.69 & 0.73 & 0.020 & 0.008 \\
\hline & Positive $(n=4) ;$ mutant type $(n=4)$ & 5.14 & 1.54 & 0.145 & 0.070 \\
\hline & All; all & 4.95 & 1.19 & 0.091 & 0.088 \\
\hline \multirow[t]{3}{*}{ Primary glioblastoma } & Negative $(n=8) ;$ wild type $(n=8)$ & 3.01 & 0.85 & 0.031 & 0.027 \\
\hline & Positive $(n=2) ;$ mutant type $(n=2)$ & 3.74 & 0.76 & 0.212 & 0.064 \\
\hline & All; all & 3.15 & 0.85 & 0.067 & 0.083 \\
\hline
\end{tabular}

NA = not applicable.

* IDH1 mutation status was detected by IHC methods and DNA sequencing. Immunostaining was interpreted as positive when tumor cell nuclei showed strong positive staining for mutant IDH1 R132H. 
diagnosis, and can also be applied for more precise identification of tumor-infiltrated margin.

2) At the same time, the detection of IDH1-mutant protein, which is based on microfluidics, can also be applied for needle biopsy samples. Because diagnostic accuracy of standard imaged-guided needle biopsy is usually hampered by an insufficient amount of sample and the limited availability of $\mathrm{IHC},{ }^{23}$ microfluidics can compensate for these disadvantages of needle biopsy.

3) A recent study of malignant astrocytomas (i.e., anaplastic astrocytoma and glioblastoma) found that $\mathrm{IDHI}$ mutant lesions are more amenable to resection. Complete resections of enhancing and nonenhancing lesions were associated with better overall outcome, suggesting the benefit of extensive resection for patients with $I D H I$ mutations. ${ }^{3}$ The study also revealed that $I D H 1$-mutant lesions displayed lower volumes of preoperative contrast enhancement but higher complete resection rates than wild-type lesions. Additionally, extensive resection in low-grade glioma predicted improved overall survival, ${ }^{28}$ suggesting a possible survival advantage. Hence, $I D H I$ mutation may also be a predictive biomarker guiding aggressive resection, in addition to an individualized therapy based on genotype. However, the current pathological technique available during surgery cannot meet the demand of intraoperative analysis. Our study results provide a reliable intraoperative technique for detection of IDHI mutation.

\section{Advantages}

Herein we describe the successful development of a new microfluidics approach for the rapid analysis of IDHI mutation from tumor tissue. The IDH1 from tiny amounts of tissue obtained by needle sampling can be analyzed within 30 minutes, with much less reagent consumption than conventional methods. Traditional enzyme-linked immunosorbent assay or IHC cannot handle samples at the sub-microgram level, and neither can these methods complete this measurement within such a short time period. Tumor samples from more than 40 patients were used to validate the proposed approach. The results from the proposed approach were also compared with those from IHC. Similar results were obtained between both approaches. The good performance, high sensitivity, and time savings can be attributed to the properties of microfluidics, especially the unique structure of the immunocolumn.

\section{Perspectives}

For the further development of microfluidics, technical improvements (such as chips that can be operated in a simpler and more automated way) are still needed. For a more accurate cutoff value between mutant and wild types, a larger-scale study should be performed using the Glioma Tissue Bank. Furthermore, because the feasibility of detecting IDHI-mutant protein has been shown, it could be used in the rapid analysis of other protein biomarkers. Therefore, further investigations should be performed to design and fabricate microfluidics, which are capable of parallel high-throughput analysis for detection of other biomarkers such as MIB-1, P53, GFAP, and so on.

\section{Conclusions}

This new approach was capable of analyzing the IDHI mutation status of tiny amounts of tissue within 30 minutes, using intraoperative microsampling. When the cutoff value was 4.11 , the sensitivity of microfluidics was $87.9 \%$ and the specificity was $64.3 \%$. In the future, based on larger sample sizes and more advanced industrialized design, it can not only supplement current techniques but also contribute to individualized therapy for improving the clinical outcomes of glioma patients.

\section{Acknowledgments}

We would like to thank all of the nurses in operating rooms and wards for their cooperation during the sample collection, Yu-qian Wang for the collection of glioma tissues, and Zhen-xiao Wang and Yan Chen for the data input of clinical information. We would also like to thank Professors Le-ming Shi and Ying Yu for their guidance in statistical analysis. Funding was received from National Key Basic Research Program of China (2013CB932502); National Natural Science Foundation of China (21377026); Shanghai Committee of Science and Technology, China (12DZ2295003); and Shanghai Municipal Health Bureau, China (XBR2011022).

\section{References}

1. Balss J, Meyer J, Mueller W, Korshunov A, Hartmann C, von Deimling A: Analysis of the IDH1 codon 132 mutation in brain tumors. Acta Neuropathol 116:597-602, 2008

2. Beebe DJ, Mensing GA, Walker GM: Physics and applications of microfluidics in biology. Annu Rev Biomed Eng 4:261-286, 2002

3. Beiko J, Suki D, Hess KR, Fox BD, Cheung V, Cabral M, et al: IDH1 mutant malignant astrocytomas are more amenable to surgical resection and have a survival benefit associated with maximal surgical resection. Neuro Oncol 16:81-91, 2014

4. Capper D, Weissert S, Balss J, Habel A, Meyer J, Jäger D, et al: Characterization of R132H mutation-specific IDH1 antibody binding in brain tumors. Brain Pathol 20:245-254, 2010

5. Capper D, Zentgraf H, Balss J, Hartmann C, von Deimling A: Monoclonal antibody specific for IDH1 R132H mutation. Acta Neuropathol 118:599-601, 2009

6. Chin CD, Laksanasopin T, Cheung YK, Steinmiller D, Linder V, Parsa H, et al: Microfluidics-based diagnostics of infectious diseases in the developing world. Nat Med 17:1015-1019, 2011

7. Choi C, Ganji SK, DeBerardinis RJ, Hatanpaa KJ, Rakheja D, Kovacs Z, et al: 2-hydroxyglutarate detection by magnetic resonance spectroscopy in IDH-mutated patients with gliomas. Nat Med 18:624-629, 2012

8. Duffy DC, McDonald JC, Schueller OJA, Whitesides GM: Rapid prototyping of microfluidic systems in poly(dimethylsiloxane). Anal Chem 70:4974-4984, 1998

9. Elkhaled A, Jalbert LE, Phillips JJ, Yoshihara HA, Parvataneni $R$, Srinivasan $R$, et al: Magnetic resonance of 2-hydroxyglutarate in IDH1-mutated low-grade gliomas. Sci Transl Med 4:116ra5, 2012

10. Felsberg J, Wolter M, Seul H, Friedensdorf B, Göppert M, Sabel MC, et al: Rapid and sensitive assessment of the IDH1 and IDH2 mutation status in cerebral gliomas based on DNA pyrosequencing. Acta Neuropathol 119:501-507, 2010

11. Gervais L, de Rooij N, Delamarche E: Microfluidic chips for point-of-care immunodiagnostics. Adv Mater 23:H151H176, 2011

12. Guo C, Pirozzi CJ, Lopez GY, Yan H: Isocitrate dehydro- 
genase mutations in gliomas: mechanisms, biomarkers and therapeutic target. Curr Opin Neurol 24:648-652, 2011

13. Horbinski C, Kofler J, Kelly LM, Murdoch GH, Nikiforova MN: Diagnostic use of IDH1/2 mutation analysis in routine clinical testing of formalin-fixed, paraffin-embedded glioma tissues. J Neuropathol Exp Neurol 68:1319-1325, 2009

14. Ichimura K, Pearson DM, Kocialkowski S, Bäcklund LM, Chan R, Jones DT, et al: IDH1 mutations are present in the majority of common adult gliomas but rare in primary glioblastomas. Neuro Oncol 11:341-347, 2009

15. Jing W, Zhao W, Liu S, Li L, Tsai CT, Fan X, et al: Microfluidic device for efficient airborne bacteria capture and enrichment. Anal Chem 85:5255-5262, 2013

16. Killela PJ, Reitman ZJ, Jiao Y, Bettegowda C, Agrawal N, Diaz LA Jr, et al: TERT promoter mutations occur frequently in gliomas and a subset of tumors derived from cells with low rates of self-renewal. Proc Natl Acad Sci U S A 110:60216026,2013

17. Lazovic J, Soto H, Piccioni D, Lou JR, Li S, Mirsadraei L, et al: Detection of 2-hydroxyglutaric acid in vivo by proton magnetic resonance spectroscopy in U87 glioma cells overexpressing isocitrate dehydrogenase-1 mutation. Neuro Oncol 14:1465-1472, 2012

18. Lee CC, Sui G, Elizarov A, Shu CJ, Shin YS, Dooley AN, et al: Multistep synthesis of a radiolabeled imaging probe using integrated microfluidics. Science 310:1793-1796, 2005

19. Louis DN, Ohgaki H, Wiestler OD, Cavenee WK, Burger PC, Jouvet A, et al: The 2007 WHO classification of tumours of the central nervous system. Acta Neuropathol 114:97-109, 2007

20. Loussouarn D, Le Loupp AG, Frenel JS, Leclair F, Von Deimling A, Aumont M, et al: Comparison of immunohistochemistry, DNA sequencing and allele-specific PCR for the detection of IDH1 mutations in gliomas. Int J Oncol 40:2058-2062, 2012

21. Ma R, de Pennington N, Hofer M, Blesing C, Stacey R: Diagnostic and prognostic markers in gliomas - an update. Br $\mathbf{J}$ Neurosurg 27:311-315, 2013

22. McDonald JC, Duffy DC, Anderson JR, Chiu DT, Wu H, Schueller OJA, et al: Fabrication of microfluidic systems in poly(dimethylsiloxane). Electrophoresis 21:27-40, 2000

23. McGirt MJ, Villavicencio AT, Bulsara KR, Friedman AH: MRI-guided stereotactic biopsy in the diagnosis of glioma: comparison of biopsy and surgical resection specimen. Surg Neurol 59:277-282, 2003

24. Meyer J, Pusch S, Balss J, Capper D, Mueller W, Christians A, et al: PCR- and restriction endonuclease-based detection of IDH1 mutations. Brain Pathol 20:298-300, 2010

25. Parsons DW, Jones S, Zhang X, Lin JC, Leary RJ, Angenendt $\mathrm{P}$, et al: An integrated genomic analysis of human glioblastoma multiforme. Science 321:1807-1812, 2008

26. Pope WB, Prins RM, Albert Thomas M, Nagarajan R, Yen $\mathrm{KE}$, Bittinger MA, et al: Non-invasive detection of 2-hydroxyglutarate and other metabolites in IDH1 mutant glioma patients using magnetic resonance spectroscopy. J Neurooncol 107:197-205, 2012

27. Preusser M, Wöhrer A, Stary S, Höftberger R, Streubel B, Hainfellner JA: Value and limitations of immunohistochemistry and gene sequencing for detection of the IDH1-R132H mutation in diffuse glioma biopsy specimens. J Neuropathol Exp Neurol 70:715-723, 2011

28. Smith JS, Chang EF, Lamborn KR, Chang SM, Prados MD, Cha $\mathrm{S}$, et al: Role of extent of resection in the long-term outcome of low-grade hemispheric gliomas. J Clin Oncol 26:1338-1345, 2008

29. Squires TM, Quake SR: Microfluidics: Fluid physics at the nanoliter scale. Rev Mod Phys 77:977-1026, 2005

30. Stone HA, Stroock AD, Ajdari A: Engineering flows in small devices: Microfluidics toward a lab-on-a-chip. Annu Rev Fluid Mech 36:381-411, 2004

31. Sui G, Wang J, Lee CC, Lu W, Lee SP, Leyton JV, et al: Solution-phase surface modification in intact poly(dimethylsiloxane) microfluidic channels. Anal Chem 78:5543-5551, 2006

32. Takano S, Tian W, Matsuda M, Yamamoto T, Ishikawa E, Kaneko MK, et al: Detection of IDH1 mutation in human gliomas: comparison of immunohistochemistry and sequencing. Brain Tumor Pathol 28:115-123, 2011

33. Whitesides GM, Ostuni E, Takayama S, Jiang X, Ingber DE: Soft lithography in biology and biochemistry. Annu Rev Biomed Eng 3:335-373, 2001

34. Yan H, Parsons DW, Jin G, McLendon R, Rasheed BA, Yuan W, et al: IDH1 and IDH2 mutations in gliomas. N Engl J Med 360:765-773, 2009

35. Zhao W, Zhang L, Jing W, Liu S, Tachibana H, Cheng X, et al: An integrated microfluidic device for rapid serodiagnosis of amebiasis. Biomicrofluidics 7:11101, 2013

\section{Disclosure}

The authors report no conflict of interest concerning the materials or methods used in this study or the findings specified in this paper.

\section{Author Contributions}

Conception and design: Aibaidula, Zhao, Chen. Acquisition of data: Aibaidula, Zhao, Chen, Shi, Zheng. Analysis and interpretation of data: Aibaidula, Zhao, Chen, Shi, Zheng. Drafting the article: Aibaidula, Zhao. Critically revising the article: Wu. Reviewed submitted version of manuscript: Wu, Aibaidula, Chen, Mao, Zhou, Sui. Approved the final version of the manuscript on behalf of all authors: Wu. Statistical analysis: Aibaidula, Shi. Administrative/technical/material support: Wu, Zhao, Mao, Zhou, Sui. Study supervision: Wu, Mao, Zhou, Sui.

\section{Supplemental Information \\ Online-Only Content}

Supplemental material is available with the online version of the article.

Supplemental Methods and Figures. http://thejns.org/doi/ suppl/10.3171/2015.4.JNS141833.

\section{Correspondence}

Jin-song Wu, Glioma Surgery Division, Neurological Surgery Department, Huashan Hospital, Shanghai Medical College, Fudan University, 12 Middle Wulumuqi Rd., Shanghai 200040, China. email:wjsongc@126.com. 